\title{
Analysis and Characterization of Staphylococcus aureus Small Colony Variants Isolated From Cystic Fibrosis Patients in Austria
}

\author{
Lilian Masoud-Landgraf ${ }^{1} \cdot$ Gernot Zarfel $^{1} \cdot$ Tanja Kaschnigg $^{1} \cdot$ Simone Friedl $^{1}$ \\ Gebhard Feierl $^{1} \cdot$ Ute Wagner-Eibel $^{1} \cdot$ Ernst Eber $^{2} \cdot$ Andrea J. Grisold $^{1}$ • \\ Clemens Kittinger ${ }^{1}$
}

Received: 18 November 2015/Accepted: 15 December 2015/Published online: 28 January 2016

(C) The Author(s) 2016. This article is published with open access at Springerlink.com

\begin{abstract}
Cystic fibrosis (CF) is the most common hereditary lung disease in the Caucasian population, characterized by viscous bronchial secretion, consecutive defective mucociliary clearance, and unavoidable colonization with microorganisms. Besides Pseudomonas aeruginosa, Staphylococcus aureus is the most common bacterial species colonizing the CF respiratory tract. Under antibiotic pressure $S$. aureus is able to switch to small colony variants (SCV). These small colony variants can invade epithelial cells, overcome antibiotic therapy inside the cells and can be the starting point for extracellular recolonization. The aim of the present study was the isolation and characterization of $S$. aureus small colony variants from Austrian cystic fibrosis patients. Samples collected from 147 patients were screened for the presence of $S$. aureus wild-type and small colony variants. Antibiotic susceptibility testing and determination of the small colony variants causing auxotrophism were performed. Wild-type isolates were assigned to corresponding small colony variants with spa typing. In total, 17 different small colony variant isolates and 12 corresponding wild-type isolates were obtained. 13 isolates were determined thymidine auxotroph, 2 isolates were auxotroph for hemin, and none of the tested isolates was auxotroph for both, respectively. The presence of SCVs is directly related to a poor clinical outcome, therefore a monitoring of SCV
\end{abstract}

Clemens Kittinger

clemens.kittinger@medunigraz.at

1 Institute of Hygiene, Microbiology and Environmental Medicine, Medical University Graz, Neue Stiftingtalstraße 2, 8010 Graz, Austria

2 Department of Paediatrics and Adolescent Medicine, Medical University of Graz, Graz, Austria prevalence is recommended. This study revealed rather low SCV ratios in CF patients compared to other countries.

\section{Introduction}

Cystic fibrosis (CF) is the most common hereditary lung disease in the white (Caucasian) population [1, 2, 13, 19, 30]. CF is caused by mutations in the gene that encodes the cystic fibrosis transmembrane conductance regulator (CFTR) protein [9, 23]. Abnormal CF transmembrane conductance regulator function initiates a pathophysiologic cascade of chronic airway inflammation and suppurative infection resulting in progressive pulmonary insufficiency. Ultimately, $95 \%$ of patients with CF succumb to respiratory failure.

In the last years, studies revealed an association between Pseudomonas aeruginosa ( $P$. aeruginosa) and CF lung function decline $[12,18]$, so the microbial researches and therapy strategies focused on $P$. aeruginosa. However, recent studies describe an increase of Staphylococcus aureus (S. aureus) prevalence in $\mathrm{CF}$, both methicillinsusceptible and methicillin-resistant (MRSA) strains [14]. Latest studies on children with CF noted similar inflammation and lung function decline during infection with $S$. aureus compared with $P$. aeruginosa [15, 28, 42].

$S$. aureus is thought to persist in CF patients over years and adapts to this environment. One occurrence of adaption is the so-called small colony variant (SCV) phenotype. This phenotype has been reported from various bacterial and fungal species and the transformation to a SCV phenotype is triggered by several stress factors $[8,20]$. The genetic background of the SCV phenotypes is based on mutations, that lead to auxotrophy in metabolic pathways. Three major types of S. aureus SCVs have been found in 
CF isolates, namely strains that are auxotroph for thymidine, menadione, or hemin [10, 41].

The switching to the SCV phenotype enables $S$. aureus to hide inside host cells and to escape from immune responses. The reduced doubling speed and alteration of pathways of SCVs also lead to an increased antibiotic resistance [3, 16, 44]. The intracellular activity of SCV phenotypes is additionally characterized by a lack of typical $S$. aureus pathogenic functions. The only persistence of SCVs does not seem to be a problem for the patient, but the instability of most SCVs bears the risk of reconversion to the fast growing and virulent wild type [25, 39]. So SCV strains have significant clinical importance and in some cases they can even persist for up to 50 years in the patient [31].

S. aureus SCVs are difficult to detect, as they generally grow slowly and often need several days to become visible on agar plates. They have altered drug-resistance profiles, such as an increased resistance to aminoglycosides [33, 36]. Currently, most clinical laboratories are unable to detect $S$. aureus SCVs, as adapted culture methods are not implemented in routine diagnostics [3, 44]. Results of susceptibility testing by disk diffusion or automated methods are often invalid or even not possible. The colonies are too small to be seen on agar plates or to be detected by automated systems [24].

Patients at risk of SCV S. aureus infection are patients with long-term antibiotic therapy [29], so besides from patients with $\mathrm{CF}$ also patients with endocarditis, pneumonia, soft tissue infections, osteomyelitis, and severe bacteraemia. The frequency of SCV recovery from clinical specimens ranges from 1 to $30 \%$ in CF patients [33].

The aim of the present study was a first epidemiological look for SCVs in the airways of CF patients in Southeast Austria. All isolates were characterized by spa typing. Additionally their antibiotic susceptibility and the underlying auxotrophism were tested, in order to provide a characterization of CF SCV S. aureus strains in our region.

\section{Materials and Methods}

Clinical isolates were obtained at the CF center at the Respiratory and Allergic Disease Division, Department of Paediatrics and Adolescent Medicine (Medical University of Graz), between January 2011 and December 2013. All CF patients of the center in Graz (ambulant and stationary patients, including all $\mathrm{CF}$ patients under treatment in Southeast Austria) are microbiologically screened at least four times per year. All S. aureus isolates included in this study were investigated at the CF Laboratory at the
Institute of Hygiene, Microbiology and Environmental Medicine (IHMEM, Medical University of Graz).

\section{Collection of Bacterial Isolates}

Sputum samples and mucoid BAL fluid samples were immediately processed with Sputasol ${ }^{\circledR}$ (Oxoid) containing the sputum liquefying agent dithiothreitol in a weight ratio of 1:2, incubated at room temperature for 10-15 min and vortexed. This was followed by a serial dilution up to a $10^{5}$ dilution. $100 \mu \mathrm{l}$ of the liquefied and diluted suspensions were plated onto TSA (Tryptocase-Soja-Agar, bioMérieux, Austria), Mac Conkey-Agar (bioMérieux, Austria), and SAID (Staphylococcus aueus identification-Agar, bioMérieux, Austria). $50 \mu \mathrm{l}$ of the liquefied origin were plated on Chocolate-Agar (bioMérieux, Austria), BCSA-Agar (bioMérieux, Austria), Schaedler-Agar (bioMérieux, Austria), and Meropenem and Polymyxin-Agar (IHMEM, Medical University Graz). All agar plates were incubated at $35^{\circ} \mathrm{C}$ for $72-120 \mathrm{~h}$ and up to 9 days at room temperature. The first screening for SCV S. aureus was carried out after $72 \mathrm{~h}$. On TSA and blood-agar, non-hemolytic, non-pigmented, small and fried egg colonies were suspected to be $S$. aureus SCVs, on SAID all colonies were suspicious to be $S$. aureus SCVs. All conspicuous colonies were inoculated on blood and Schaedler-Agar [21]. If the colonies were normal-sized hemolytic and pigmented on Schaedler-Agar and not on blood-agar, they were considered to be $S$. aureus SCVs. These isolates were tested for species identification by MALDI-TOF MS Axima ${ }^{\mathrm{TM}}$ Assurance (Shimadzu, Japan). For this purpose, one colony of each isolate was directly spotted on the FlexiMass MALDI target plate. For automatic measurement and identification the SARAMIS $^{\text {TM }}$ (spectral archived microbial identification system) database application from bioMérieux was applied. Isolation of all other bacterial species was done as described previously [26].

\section{Spa Typing}

Spa typing is based on sequencing the polymorphic X-region of the protein A gene (spa). This is a well-established technique and the results can be compared with databases. The determined spa types can be assigned to distinct groups with known origin and characteristics [10].

\section{Detection of $m e c$ A Gene}

To confirm MRSA genotype, the presence of mecA was analyzed in all isolates with the BD $\mathrm{MAX}^{\mathrm{TM}}$ System (Beckton Dickinson Austria, Vienna), using the "BD MAX MRSA assay" according to the manufacturer's instructions. 


\section{Auxotrophism Test}

SCV auxotrophism was tested using agar disk diffusion test according to Maduka-Ezeh et al. on Mueller-Hinton agar for thymidine, hemin, and menadione [37]. To identify potentially double auxotrophic strains, the agar plates were also loaded with all three test substrates additionally [4, 32, 35, 38].

\section{Determination of Antibiotic Resistance}

Antibiotics tested were penicillin, oxacillin, gentamicin, erythromycin, clindamycin, ciprofloxacin, tetracycline, rifampicin, fusidic acid, vancomycin, linezolid, and mupirocin. Resistance testing was performed as recommended by the European Committee on Antimicrobial Susceptibility Testing (EUCAST, http://www.eucast.org/clinical_breakpoints/).

For the normal $S$. aureus strains, determination of the minimal inhibitory concentration (MIC) was performed by using VITEK-2 (bioMérieux, Solna, Sweden) and disk diffusion method, and by E-test (bioMérieux, Solna, Sweden). For the determination of the antimicrobial susceptibilities of the SCV S. aureus strains resistance was tested on brain-heart infusion (BHI) agar [21].

\section{Results}

\section{Collection of Bacterial Isolates}

Samples originated from 147 patients ( 75 male, 72 female). The patients' age ranged from 10 months to 56 years, with a median age of 19 years. Samples included sputa $(n=1452)$, nasal swabs ( $n=518)$, throat swabs $(n=466)$, and bronchoalveolar lavage (BAL) fluids $(\mathrm{n}=58)$. In total, $1399 \mathrm{~S}$. aureus isolates could be recovered in 127 patients (86.4\%), two of them were tested phenotypically and genotypically (mecA gene) positive for MRSA. 12 patients harbored SCV $S$. aureus isolates including the two MRSA positive patients. So, $8.2 \%$ of the CF patients and $9.4 \%$ of $S$. aureus positive $\mathrm{CF}$ patients were positive for $S$. aureus $\mathrm{SCV}$, respectively. 74 S. aureus (27 SCV and 47 wild types) could be isolated from the $12 \mathrm{SCV}$ positive patients during the study period.

Only eight $(66.7 \%)$ of the patients with SCV strains were co-colonized with $P$. aeruginosa, whereas 95 of 127 (74.8\%) of $S$. aureus positive patients were also positive for $P$. aeruginosa. None of them was co-colonized with Haemophilus influenzae and three $(18.8 \%)$ of them were positive for Stenotrophomonas maltophilia.

\section{Spa Typing}

In total, 21 different spa types could be detected. SCV $S$. aureus revealed 13 spa types; 10 of these spa types could also be identified in wild-type $S$. aureus isolates from the same patient (Table 1). Three SCV spa types could not be assigned to a wild-type strain and 8 spa types were only present in wild type (data not shown). Only two spa types (t002 and t085) were present in more than one patient (Table 1).

S. aureus SCV were classified as identical if they had the same spa type and the same auxotrophism. 10 out of 12 CF patients (patients 1, 2, 3, 4, 6, 7, 8, 10, 11, and 12) harbored one SCV isolate. From two patients (patients 5 and 9) SCV S. aureus with different wild-type strains could be isolated. The isolated strain of patient 9 was the only one that had developed different SCV phenotypes (Table 1).

\section{Auxotrophism}

Thirteen of the tested 17 isolates showed thymidine auxotrophism, and two times hemin dependence was recovered; menadione auxotrophism could not be linked to any isolate. Two isolates showed no response to the substrates and the auxotrophism could therefore not be addressed (Table 1). In contrast to the others, the t085 isolates of patient 9 showed SCV phenotype differentiation into thymidine, hemin, and a non-assignable type of auxotrophism. This spa type was also the only one that had developed different SCV phenotypes.

\section{Antibiotic Susceptibility of SCVs}

Antibiotic susceptibility was determined for 17 strains. The highest resistance rate was observed for penicillin/ampicillin with 14 isolates $(82.4 \%)$. Resistance against erythromycin/clindamycin was present in nine isolates $(52.9 \%)$. On the basis of MIC testing with E-test, two isolates $(11.7 \%)$ were oxacillin resistant and therefore MRSA. All tested isolates were susceptible to tetracyclin, fusidic acid, linezolid, vancomycin, and mupirocin (Table 2).

\section{Discussion}

Over a period of long-term infection, S. aureus may undergo extensive adaptation to the lung environment in CF patients [17]. One mentionable effect of this adaptation is the transition toward a slow-growing low virulence state, the small colony variant. SCVs mutants enable advantages under chronic infection conditions. For example, SCVs have the ability to survive intracellularly, where they are protected against phagocytosis and from extracellular antimicrobial agents. So, they overcome different environmental pressures (antibiotic treatment or bacterial 
Table 1 Antibiotic resistance pattern, type of auxotrophism, and spa types of the SCV isolates of 12 patients

\begin{tabular}{|c|c|c|c|c|c|c|}
\hline SCV & Pat $\mathrm{Nr}$ & $\mathrm{SCV}$ isolation date & Auxotrophism & $\begin{array}{l}\text { Spa- } \\
\text { type }\end{array}$ & Resistance & Isolation date of the paired $S$. aureus strain \\
\hline 1 & 1 & 28.02 .2013 & Thymidine & $\mathrm{t} 12308$ & $\mathrm{P}, \mathrm{AM}, \mathrm{E}, \mathrm{CC}, \mathrm{GM}$ & $\begin{array}{l}28.05 .2010 \\
15.12 .2010 \\
05.11 .2013\end{array}$ \\
\hline 2 & 2 & 07.02 .2013 & Thymidine & $\mathrm{t} 209$ & $\mathrm{P}, \mathrm{AM}, \mathrm{E}, \mathrm{CC}$ & $\begin{array}{l}28.08 .2010 \\
07.12 .2011 \\
29.06 .2012\end{array}$ \\
\hline 3 & 3 & 25.02 .2013 & Thymidine & t012 & $\mathrm{P}, \mathrm{AM}$ & Paired strain could not be found \\
\hline 4 & 4 & 17.10 .2013 & Thymidine & $\mathrm{t} 732$ & $\mathrm{E}, \mathrm{CC}$ & 04.10 .2010 \\
\hline 5 & 5 & 07.11 .2013 & Thymidine & t002 & $\mathrm{OXA}, \mathrm{P}, \mathrm{AM}, \mathrm{E}, \mathrm{CC}, \mathrm{GM}, \mathrm{NN}$ & $\begin{array}{l}28.11 .2012 \\
07.11 .2013\end{array}$ \\
\hline 6 & 5 & 30.08 .2012 & Thymidine & $\mathrm{t} 355$ & OXA, P, AM, GM, RIF & $\begin{array}{l}14.04 .2010 \\
07.07 .2010 \\
02.11 .2010 \\
21.02 .2011\end{array}$ \\
\hline 7 & 6 & 08.07 .2013 & Thymidine & $\mathrm{t} 8012$ & $\mathrm{P}, \mathrm{AM}, \mathrm{E}, \mathrm{CC}$ & $\begin{array}{l}20.07 .2010 \\
28.10 .2013\end{array}$ \\
\hline 8 & 7 & 11.09.2012 & Thymidine & t004 & GM & $\begin{array}{l}25.07 .2011 \\
30.07 .2013\end{array}$ \\
\hline 9 & 8 & 11.04 .2013 & Thymidine & t085 & $\mathrm{P}, \mathrm{AM}$ & Paired strain could not be found \\
\hline 10 & 9 & 08.08 .2012 & Thymidine & $\mathrm{t} 085$ & $\mathrm{P}, \mathrm{AM}$ & 09.05 .2012 \\
\hline 11 & 9 & 16.03 .2013 & Not known & t085 & & \\
\hline 12 & 9 & 15.05 .2013 & Hemin & t085 & $\mathrm{P}, \mathrm{AM}, \mathrm{GM}$ & \\
\hline 13 & 9 & 20.09.2011 & Thymidine & t645 & $\mathrm{P}, \mathrm{AM}, \mathrm{E}, \mathrm{CC}, \mathrm{GM}$ & Paired strain could not be found \\
\hline 14 & 9 & 13.02 .2013 & Thymidine & t015 & $\mathrm{P}, \mathrm{AM}, \mathrm{CIP}$ & $\begin{array}{l}25.05 .2010 \\
25.01 .2011\end{array}$ \\
\hline 15 & 10 & 25.06 .2013 & Not known & $\mathrm{t} 2249$ & $\mathrm{P}, \mathrm{AM}, \mathrm{E}, \mathrm{CC}, \mathrm{GM}$ & $\begin{array}{l}02.11 .2010 \\
17.10 .2012 \\
16.07 .2013\end{array}$ \\
\hline 16 & 11 & 05.09 .2013 & Hemin & t002 & $\mathrm{P}, \mathrm{AM}, \mathrm{E}, \mathrm{CC}$ & 10.08 .2010 \\
\hline 17 & 12 & 12.09 .2012 & Thymidine & t9847 & $\mathrm{P}, \mathrm{AM}, \mathrm{E}, \mathrm{CC}$ & $\begin{array}{l}12.07 .2010 \\
06.11 .2012 \\
20.11 .2013\end{array}$ \\
\hline
\end{tabular}

OXA oxacillin, $P$ penicillin, $A M$ ampicillin, $E$ erythromycin, $C C$ clindamycin, $G M$ gentamicin, $N N$ tobramycin, $C I P$ ciprofloxacin, $R I F$ rifampicin

competitors). The relation between the presence of SCVs and a poor clinical outcome has already been reported in several studies, with patients harboring SCV variants having lower blood oxygen levels and a significantly lower FEV1 score [5, 34].

Long-term treatment with trimethoprim/sulfamethoxazole (SXT) or aminoglycosides favors the emergence of thymidine-dependent SCVs in CF patients [7, 43]. This is in concordance with our data, as the patients in our study had previously been treated with SXT.

The prevalence of thymidine-dependent SCV strains in CF patients has already been described [6, 11, 22]. We also found a majority of thymidine-dependent strains, whereas not a single menadione-dependent strain could be detected. The reason for the absence of menadione auxotroph SCVs might be the extremely slow growth of the menadione auxotroph SCVs which could have been overgrown by faster growing variants.

Recent publications e.g., Melter et al. reported that SCV variants are much more resistant to antibiotics than the non-SCV S. aureus isolates [29]. In contrast, we could not find any difference in the resistance rates between wildtype S. aureus strains and SCV S. aureus strains.

Twelve patients were SCV positive, so the SCV $S$. aureus prevalence was $8.1 \%$, lower than previously reported from $\mathrm{CF}$ studies in other countries [45]. The 
Table 2 Antibiotic resistance within the SCV isolates

\begin{tabular}{lllll}
\hline Antibiotic & Nr. of S & $\% \mathrm{~S}$ & $\mathrm{Nr}$. of R & $\% \mathrm{R}$ \\
\hline Ampicillin & 3 & 17.6 & 14 & 82.4 \\
Penicillin & 3 & 17.6 & 14 & 82.4 \\
Oxacillin & 15 & 88.3 & 2 & 11.7 \\
Amoxicillin/clavulanic acid & 15 & 88,3 & 2 & 11.7 \\
Piperacillin/tazobactam & 15 & 88.3 & 2 & 11.7 \\
Gentamicin & 10 & 58.8 & 7 & 41,2 \\
Tetracyclin & 17 & 100 & 0 & 0 \\
Ciprofloxacin & 16 & 94.1 & 1 & 5.9 \\
Erythromycin & 8 & 47.1 & 9 & 52.9 \\
Clindamycin & 8 & 47.1 & 9 & 52.9 \\
Fusidic acid & 17 & 100 & 0 & 0 \\
Rifampicin & 16 & 94.1 & 0 & 5.9 \\
Linezolid & 17 & 100 & 0 & 0 \\
Vamcomycin & 17 & 100 & 0 & 0 \\
Mupirocin & 17 & 100 & 0 & 0 \\
\hline
\end{tabular}

$N r . S$ number of sensitive isolates, $\% S$ percentage of sensitive isolates, $N r$ of $R$ number of resistant isolates, $\% R$ percentage of resistant isolates

prevalence of MRSA also is very low in our center. Oxacillin resistance was found in $11.7 \%$ of the SCVs. This could be related to local infection control and the low incidence of nosocomial $S$. aureus acquisition in our $\mathrm{CF}$ center; multiple antibiotic-resistant $S$. aureus $\mathrm{SCV}$ s was not a common occurrence in this study [27, 43].

The suggested inductive effect on $S$. aureus SCV via $P$. aeruginosa co-infection could not be verified in our study but this might be related to the relatively low number of $S$. aureus SCV isolates [40, 43].

In conclusion, this first report on $S$. aureus SCVs from an Austrian CF center revealed a high number of $S$. aureus positive $\mathrm{CF}$ patients but rather low SCV prevalence. A tight cooperation between clinicians and microbiological laboratory is essential for a successful management of the underestimated SCV challenge.

\section{Acknowledgments Open access funding provided by Medical} University of Graz.

\section{Compliance with Ethical Standards}

Conflict of Interest The authors declare that they have no conflict of interest.

Open Access This article is distributed under the terms of the Creative Commons Attribution 4.0 International License (http://crea tivecommons.org/licenses/by/4.0/), which permits unrestricted use, distribution, and reproduction in any medium, provided you give appropriate credit to the original author(s) and the source, provide a link to the Creative Commons license, and indicate if changes were made.

\section{References}

1. Accurso FJ (2008) Update in cystic fibrosis 2007. Am J Respir Crit Care Med 177:1058-1061. doi:10.1164/rccm.200801-069UP

2. Accurso FJ (2007) Update in cystic fibrosis 2006. Am J Respir Crit Care Med 175:754-757. doi:10.1164/rccm.200701-160UP

3. Balke B, Hoy L, Weissbrodt H, Haussler S (2004) Comparison of the Micronaut Merlin automated broth microtiter system with the standard agar dilution method for antimicrobial susceptibility testing of mucoid and nonmucoid Pseudomonas aeruginosa isolates from cystic fibrosis patients. Eur J Clin Microbiol Infect Dis 23:765-771. doi:10.1007/s10096-004-1212-7

4. Ballarini A, Scalet G, Kos M, Cramer N, Wiehlmann L, Jousson O (2012) Molecular typing and epidemiological investigation of clinical populations of Pseudomonas aeruginosa using an oligonucleotide-microarray. BMC Microbiol 12:152. doi:10. 1186/1471-2180-12-152

5. Bell SC, Bowerman AM, Nixon LE, Macdonald IA, Elborn JS, Shale DJ (2000) Metabolic and inflammatory responses to pulmonary exacerbation in adults with cystic fibrosis. Eur J Clin Invest 30:553-559. doi:10.1046/j.1365-2362.2000.00667.x

6. Besier S, Smaczny C, von Mallinckrodt C, Krahl A, Ackermann H, Brade V, Wichelhaus TA (2007) Prevalence and clinical significance of Staphylococcus aureus small-colony variants in cystic fibrosis lung disease. J Clin Microbiol 45:168-172. doi:10. 1128/JCM.01510-06

7. Chatterjee I, Kriegeskorte A, Fischer A, Deiwick S, Theimann N, Proctor RA, Peters G, Herrmann M, Kahl BC (2008) In vivo mutations of thymidylate synthase (encoded by thyA) are responsible for thymidine dependency in clinical small-colony variants of Staphylococcus aureus. J Bacteriol 190:834-842. doi: 10.1128/JB.00912-07

8. Chen XJ, Clark-Walker GD (2000) The petite mutation in yeasts: 50 years on. Int Rev Cytol 194:197-238

9. Doring G, Gulbins E (2009) Cystic fibrosis and innate immunity: how chloride channel mutations provoke lung disease. Cell Microbiol 11:208-216. doi:10.1111/j.1462-5822.2008.01271.x

10. Feil EJ, Li BC, Aanensen DM, Hanage WP, Spratt BG (2004) eBURST: inferring patterns of evolutionary descent among clusters of related bacterial genotypes from multilocus sequence typing data. J Bacteriol 186:1518-1530

11. Gilligan PH, Gage PA, Welch DF, Muszynski MJ, Wait KR (1987) Prevalence of thymidine-dependent Staphylococcus aureus in patients with cystic fibrosis. J Clin Microbiol 25:1258-1261

12. Gomez MI, Prince A (2007) Opportunistic infections in lung disease: Pseudomonas infections in cystic fibrosis. Curr Opin Pharmacol 7:244-251. doi:10.1016/j.coph.2006.12.005

13. Goss CH, Ratjen F (2013) Update in cystic fibrosis 2012. Am J Respir Crit Care Med 187:915-919. doi:10.1164/rccm.2013010184UP

14. Govan JR (2000) Infection control in cystic fibrosis: methicillinresistant Staphylococcus aureus, Pseudomonas aeruginosa and the Burkholderia cepacia complex. J R Soc Med 93(Suppl 38):40-45

15. Govan JR, Deretic V (1996) Microbial pathogenesis in cystic fibrosis: mucoid Pseudomonas aeruginosa and Burkholderia cepacia. Microbiol Rev 60:539-574

16. Haussler S, Ziesing S, Rademacher G, Hoy L, Weissbrodt H (2003) Evaluation of the Merlin, Micronaut system for automated antimicrobial susceptibility testing of Pseudomonas aeruginosa and Burkholderia species isolated from cystic fibrosis patients. Eur J Clin Microbiol Infect Dis 22:496-500. doi:10.1007/s10096003-0974-7

17. Hirschhausen N, Block D, Bianconi I, Bragonzi A, Birtel J, Lee JC, Dubbers A, Kuster P, Kahl J, Peters G et al (2013) Extended 
Staphylococcus aureus persistence in cystic fibrosis is associated with bacterial adaptation. Int J Med Microbiol 303:685-692. doi:10.1016/j.ijmm.2013.09.012

18. Hogardt M, Ulrich J, Riehn-Kopp H, Tummler B (2009) EuroCareCF quality assessment of diagnostic microbiology of cystic fibrosis isolates. J Clin Microbiol 47:3435-3438. doi:10.1128/ JCM.01182-09

19. Jain M, Goss CH (2014) Update in cystic fibrosis 2013. Am J Respir Crit Care Med 189:1181-1186. doi:10.1164/rccm.2014020203UP

20. Johns BE, Purdy KJ, Tucker NP, Maddocks SE (2015) Phenotypic and genotypic characteristics of small colony variants and their role in chronic infection. Microbiol Insights 8:15-23. doi:10.4137/MBI.S25800

21. Kahl B, Herrmann M, Everding AS, Koch HG, Becker K, Harms E, Proctor RA, Peters G (1998) Persistent infection with small colony variant strains of Staphylococcus aureus in patients with cystic fibrosis. J Infect Dis 177:1023-1029

22. Kahl B, Herrmann M, Everding AS, Koch HG, Becker K, Harms E, Proctor RA, Peters G (1998) Persistent infection with small colony variant strains of Staphylococcus aureus in patients with cystic fibrosis. J Infect Dis 177:1023-1029

23. Kerem E, Corey M, Kerem BS, Rommens J, Markiewicz D, Levison H, Tsui LC, Durie P (1990) The relation between genotype and phenotype in cystic fibrosis-analysis of the most common mutation (delta F508). N Engl J Med 323:1517-1522. doi:10.1056/NEJM199011293232203

24. Looney WJ (2000) Small-colony variants of Staphylococcus aureus. Br J Biomed Sci 57:317-322

25. Maiden MC (2006) Multilocus sequence typing of bacteria. Annu Rev Microbiol 60:561-588. doi:10.1146/annurev.micro.59.030804.121 325

26. Masoud-Landgraf L, Badura A, Eber E, Feierl G, Posch J, Zarfel G, Zach M, Marth E (2012) Molecular epidemiology of Pseudomonas aeruginosa in cystic fibrosis patients from Southeast Austria. Wien Klin Wochenschr 124:262-265. doi:10.1007/ s00508-012-0156-7

27. Masoud-Landgraf L, Johler S, Badura A, Feierl G, Luxner J, Wagner-Eibel U, Eber E, Zarfel G, Grisold AJ (2015) Genetic and phenotypic characteristics of staphylococcus aureus isolates from cystic fibrosis patients in Austria. Respiration 89:390-395. doi: $10.1159 / 000377707$

28. Mathee K, Narasimhan G, Valdes C, Qiu X, Matewish JM, Koehrsen M, Rokas A, Yandava CN, Engels R, Zeng E et al (2008) Dynamics of Pseudomonas aeruginosa genome evolution. Proc Natl Acad Sci U S A 105:3100-3105. doi:10.1073/pnas. 0711982105

29. Melter O, Radojevic B (2010) Small colony variants of Staphylococcus aureus—review. Folia Microbiol (Praha) 55:548-558. doi:10.1007/s12223-010-0089-3

30. O'Sullivan BP, Freedman SD (2009) Cystic fibrosis. Lancet 373:1891-1904. doi:10.1016/S0140-6736(09)60327-5

31. Pirnay JP, Bilocq F, Pot B, Cornelis P, Zizi M, Van Eldere J, Deschaght P, Vaneechoutte M, Jennes S, Pitt T et al (2009) Pseudomonas aeruginosa population structure revisited. PLoS ONE 4:e7740. doi:10.1371/journal.pone.0007740

32. Rakhimova E, Wiehlmann L, Brauer AL, Sethi S, Murphy TF, Tummler B (2009) Pseudomonas aeruginosa population biology in chronic obstructive pulmonary disease. J Infect Dis 200:19281935. doi: $10.1086 / 648404$
33. Romling U, Wingender J, Muller H, Tummler B (1994) A major Pseudomonas aeruginosa clone common to patients and aquatic habitats. Appl Environ Microbiol 60:1734-1738

34. Schneider M, Muhlemann K, Droz S, Couzinet S, Casaulta C, Zimmerli S (2008) Clinical characteristics associated with isolation of small-colony variants of Staphylococcus aureus and Pseudomonas aeruginosa from respiratory secretions of patients with cystic fibrosis. J Clin Microbiol 46:1832-1834. doi:10.1128/ JCM.00361-08

35. Selezska K, Kazmierczak M, Musken M, Garbe J, Schobert M, Haussler S, Wiehlmann L, Rohde C, Sikorski J (2012) Pseudomonas aeruginosa population structure revisited under environmental focus: impact of water quality and phage pressure. Environ Microbiol 14:1952-1967. doi:10.1111/j.1462-2920. 2012.02719.x

36. Speert DP, Campbell ME, Henry DA, Milner R, Taha F, Gravelle A, Davidson AG, Wong LT, Mahenthiralingam E (2002) Epidemiology of Pseudomonas aeruginosa in cystic fibrosis in British Columbia, Canada. Am J Respir Crit Care Med 166:988-993

37. Tubbs D, Lenney W, Alcock P, Campbell CA, Gray J, Pantin C (2001) Pseudomonas aeruginosa in cystic fibrosis: cross-infection and the need for segregation. Respir Med 95:147-152. doi:10.1053/rmed.2000.1009

38. Tummler B, Wiehlmann L, Klockgether J, Cramer N (2014) Advances in understanding Pseudomonas. F1000Prime Rep 6:9. doi:10.12703/P6-9

39. Turner KM, Hanage WP, Fraser C, Connor TR, Spratt BG (2007) Assessing the reliability of eBURST using simulated populations with known ancestry. BMC Microbiol 7:30. doi:10.1186/14712180-7-30

40. Vergison A, Denis O, Deplano A, Casimir G, Claeys G, DeBaets F, DeBoeck K, Douat N, Franckx H, Gigi J et al (2007) National survey of molecular epidemiology of Staphylococcus aureus colonization in Belgian cystic fibrosis patients. J Antimicrob Chemother 59:893-899. doi:10.1093/jac/dkm037

41. Wiehlmann L, Wagner G, Cramer N, Siebert B, Gudowius P, Morales G, Kohler T, van Delden C, Weinel C, Slickers P et al (2007) Population structure of Pseudomonas aeruginosa. Proc Natl Acad Sci U S A 104:8101-8106. doi:10.1073/pnas.06092 13104

42. Wolfgang MC, Kulasekara BR, Liang X, Boyd D, Wu K, Yang Q, Miyada CG, Lory S (2003) Conservation of genome content and virulence determinants among clinical and environmental isolates of Pseudomonas aeruginosa. Proc Natl Acad Sci U S A 100:8484-8489. doi:10.1073/pnas.0832438100

43. Wolter DJ, Emerson JC, McNamara S, Buccat AM, Qin X, Cochrane E, Houston LS, Rogers GB, Marsh P, Prehar K et al (2013) Staphylococcus aureus small-colony variants are independently associated with worse lung disease in children with cystic fibrosis. Clin Infect Dis 57:384-391. doi:10.1093/cid/ cit270

44. Wolter JM, Kotsiou G, McCormack JG (1995) Mixed morphotype testing of Pseudomonas aeruginosa cultures from cystic fibrosis patients. J Med Microbiol 42:220-224

45. Yagci S, Hascelik G, Dogru D, Ozcelik U, Sener B (2013) Prevalence and genetic diversity of Staphylococcus aureus smallcolony variants in cystic fibrosis patients. Clin Microbiol Infect 19:77-84. doi:10.1111/j.1469-0691.2011.03742.x 